\title{
Optimal Information Design for Search Goods
}

\author{
By Michael Choi, Kyungmin Kim, Marilyn Pease*
}

The fast-growing literature on information design offers novel insights into many economic problems. Its impact has been particularly strong in the field of industrial organization. Information design has deepened our understanding of monopoly pricing (Roesler and Szentes 2017), price discrimination (Bergemann, Books, and Morris 2015), auction design (Bergemann and Pesendorfer 2007; Bergemann, Brooks, and Morris 2017), advertising (Anderson and Renault 2006), and refunds (Hinnosaar and Kawai 2018), to name a few.

Most studies, however, consider experience goods (whose true values are revealed only through consumption), and there has been little work on search goods (whese true values are available upon inspection). 1 The only exception we are aware of is Anderson and Renault (2006), who study the monopoly problem for search goods with advertising..$^{2}$ This asymmetry is in part because search goods present a unique technical challenge. With experience goods, a (risk-neutral) consumer makes a purchase decision only based on her conditional expectation. Therefore, the economic effects of information provision can be directly assessed through its effects on the distribution of conditional expectations. With search goods, however, a consumer

\footnotetext{
*Choi: Department of Economics, University of California, Irvine, 3151 Social Science Plaza, Irvine, CA 92697 (email: michael.yfchoi@uci.edu); Kim: Department of Economics, University of Miami, 5250 University Drive, Jenkins Bldg \#521, Coral Gables, FL 33146 (email: kkim@ bus.miami.edu); Pease: Department of Business Economics and Public Policy, Kelley School of Business, Indiana University, 1275 E. 10th Street, Bloomington, IN 47405 (email: marpease@iu.edu).

${ }^{\dagger}$ Go to https://doi.org/10.1257/pandp.20191101 to visit the article page for additional materials and author disclosure statement(s).

${ }^{1}$ This fundamental distinction between experience goods and search goods is due to Nelson (1970).

${ }^{2}$ Several recent papers (e.g., Au 2018; Hu and Dogan 2018; Hulko and Whitmeyer 2018) study an information design problem in a search environment. However, to our knowledge, all of them still consider experience goods.
}

uses the provided information to make a search (visit) decision, but necessarily relies on her true value for her purchase decision. Therefore, the economic role of information is indirect and more subtle. ${ }^{3}$

As one step toward filling this gap, we consider the basic monopoly problem for search goods. Given an information structure (signal), the seller sets a price. Then, a (representative) consumer observes a signal realization and the price, and decides whether to visit the seller or not. If the consumer visits, then she learns her true value (either 0 or 1 ) and decides whether to purchase or not. In the same environment, Anderson and Renault (2006) provide a general characterization for the seller-optimal signal. We focus on the consumer-optimal signal (and the seller-worst signal).

Our model can be interpreted as a search-good counterpart to Roesler and Szentes (2017). As in their paper, unit-elastic demand plays an important role in our analysis. However, there are three notable differences. First, unit elasticity is generated by the conditional distribution given the high value, not by the unconditional distribution. Second, the consumer-optimal signal and the seller-worst signal, which always coincide in Roesler and Szentes (2017), may differ depending on search costs. Finally, neither the seller-worst signal nor the consumer-optimal signal yields efficient trade. All of these differences are precisely due to the nature of search goods: a consumer's search decision depends on available information, but her purchase decision is based on her true value.

\footnotetext{
${ }^{3} \mathrm{~A}$ similar problem arises with an experience good if a consumer can return the good after purchase. Hinnosaar and Kawai (2018) study this problem and characterize the set of all feasible outcomes when the seller can optimally set a refund policy.
} 


\section{The Model}

A seller supplies a product at zero marginal cost. A (representative) consumer is uncertain about the product's value $v$ to her, which can be either 0 or 1 . It is common knowledge that the ex ante probability that $v=1$ is equal to $\mu_{0} \in(0,1)$. The timing of the game is as follows. First, the seller sets a price $p$. Then, the consumer receives a signal $s$ about her true value $v$ according to a function $\pi:\{0,1\} \times S \rightarrow[0,1]$, where $\pi(v, s)$ denotes the probability that the consumer observes $s$ conditional on true value $v$. The information structure $\pi$ is known to both players. Given $p$ and $s$, the consumer decides whether to visit the seller or not; she must visit in order to purchase. If the consumer visits, she pays $c(>0) \cdot{ }^{4}$ For expositional simplicity, we focus on the case where $c<\mu_{0}$. If the consumer pays $c$, then she learns her true value, regardless of her prior information, and decides whether to purchase or not. If trade occurs, then the seller gets a payoff of $p$ and the consumer receives a payoff of $v-p$. If not, both players get payoffs of 0 . Both players are risk neutral and maximize their expected utility.

As is well-known, the information structure can be conveniently reformulated in terms of the distribution of posteriors. Specifically, each function $\pi$ induces a particular distribution $G$ of posteriors $\mu=\operatorname{Pr}\{v=1 \mid s\}$. Conversely, for any Bayes-plausible distribution $G$ (i.e., $\left.\int \mu d G(\mu)=\mu_{0}\right)$, there exists a signal function $\pi$ that yields $G$. In what follows, we directly refer to $G$ as a signal.

We seek to characterize the consumeroptimal signal (that maximizes consumer surplus) and the seller-worst signal (that minimizes the seller's expected profit). 5 To formally define consumer surplus and profit, observe that given

\footnotetext{
${ }^{4}$ In the literature, this visit (search) cost is typically interpreted as either the transportation cost of visiting the seller or the cost of inspecting the product. In our environment where the consumer's information varies, while $c$ is fixed, the former (transportation-cost) interpretation is more suitable than the latter (inspection-cost) interpretation.

${ }^{5}$ In our environment, the seller-optimal signal is perfectly informative, which enables the seller to entice the consumer to visit only when her true value is 1 and charge the highest possible price $1-c$. Since this leaves no surplus to the consumer, it is also a consumer-worst signal. See Anderson and Renault (2006) for a general characterization of the seller-optimal signal.
}

posterior $\mu$ and price $p$, the consumer visits if and only if

$$
\mu(1-p)-c \geq 0 \Leftrightarrow \mu \geq \underline{\mu}(p) \equiv \frac{c}{1-p} .
$$

In addition, since each consumer discovers her true value upon visit, she purchases only when $v \geq p$, which holds if and only if $v=1$ for $p \in(0,1)$. These conditions imply that the consumer's expected payoff and the probability that she purchases the product are, respectively, equal to

$$
v_{B}(\mu)=\max \{0, \mu(1-p)-c\}
$$

and

$$
D(\mu)=\left\{\begin{array}{ll}
0 & \text { if } \mu<\underline{\mu}(p) \\
\mu & \text { if } \mu \geq \underline{\mu}(p)
\end{array} .\right.
$$

Consumer surplus and profit (producer surplus) then are defined as follows:

$$
C S(G, p)=\int_{\underline{\mu}}^{1}(p)(\mu(1-p)-c) d G(\mu)
$$

and

$$
P S(G, p)=p \int_{\underline{\mu}(p)}^{1} \mu d G(\mu) .
$$

\section{Optimal Information Design}

\section{A. Feasible Conditional Distributions}

Since the consumer's search decision is based on belief $\mu$, while her purchase decision depends on true value $v$, it is convenient to define the following conditional distributions: for each $v \in\{0,1\}$, let $G_{v}$ denote the distribution of posteriors conditional on $v$. In other words, $G_{v}(\mu)$ is the probability that the consumer's belief is below $\mu$ when her true value is $v$. Given $G$, both $G_{0}$ and $G_{1}$ can be uniquely derived through the following two consistency requirements: for all $\mu \in[0,1]$,

$$
G(\mu)=\left(1-\mu_{0}\right) G_{0}(\mu)+\mu_{0} G_{1}(\mu)
$$

and 
(2)

$$
\text { 2) } \begin{aligned}
\mu & =\frac{\mu_{0} d G_{1}(\mu)}{\mu_{0} d G_{1}(\mu)+\left(1-\mu_{0}\right) d G_{0}(\mu)} \Leftrightarrow \\
\frac{\mu}{1-\mu} & =\frac{\mu_{0}}{1-\mu_{0}} \frac{d G_{1}(\mu)}{d G_{0}(\mu)} .
\end{aligned}
$$

The former simply states that the unconditional distribution is a weighted sum of the conditional distributions, while the latter is Bayes' rule.

Since the seller is concerned only with eventual purchase (that is, consumers who visit and purchase), $G_{1}$ plays an important role in the subsequent analysis. The following lemma provides a simple necessary and sufficient condition for the feasibility of $G_{1}$.

LEMMA 1: A distribution function $G_{1}$ is feasible (i.e., there exists a feasible unconditional distribution function $G$ that generates the conditional distribution function $G_{1}$ ) if and only if

(3) $\Phi\left(G_{1}\right) \equiv 1-\frac{\mu_{0}}{1-\mu_{0}} \int \frac{1-\mu}{\mu} d G_{1}(\mu) \geq 0$.

If $G_{1}$ first-order-stochastically dominates $G_{1}^{\prime}$, then $\Phi\left(G_{1}\right) \geq \Phi\left(G_{1}^{\prime}\right)$.

\section{PROOF:}

Given $G_{1}, G_{0}$ is uniquely determined by (2). By construction, equation (1) always holds and $G_{0}$ is weakly increasing. Therefore, for the feasibility of $G_{1}$, it is necessary and sufficient that

$$
\begin{aligned}
\Phi\left(G_{1}\right) & \equiv G_{0}(0)=1-\int_{0+}^{1} d G_{0}(\mu) \\
& =1-\frac{\mu_{0}}{1-\mu_{0}} \int \frac{1-\mu}{\mu} d G_{1}(\mu) \geq 0 .
\end{aligned}
$$

The last claim is true because $(1-\mu) / \mu$ falls in $\mu$ and thus the integral in equation (3) decreases as $G_{1}$ increases in the sense of first-order stochastic dominance.

Intuitively, in order to induce posterior $\mu$, the measures (densities) of high- and low-value consumers need to be balanced in such a way that Bayes' rule (2) holds. The only exception is when $\mu=0$, as this posterior is induced whenever $d G_{1}(\mu) / d G_{0}(\mu)=0$. Therefore, for the feasibility of $G_{1}$, it is necessary and sufficient that the corresponding distribution $G_{0}$ is such that $G_{0}(0)=\Phi\left(G_{1}\right) \geq 0$. In addition, if $G_{1}$ increases in the sense of first-order stochastic dominance, then the mass of $v=0$ receiving a signal larger than 0 decreases, so that $G_{0}(0)=\Phi\left(G_{1}\right)$ increases.

\section{B. Unit-Elastic Distributions}

The following class of conditional distributions plays a crucial role in determining the seller-worst and consumer-optimal signals. ${ }^{6}$

DEFINITION 1: For $\underline{\mu} \in(0,1)$ and $\bar{\mu}(\geq \underline{\mu})$, let $G_{\overline{1}}^{\mu, \bar{\mu}}$ be a distribution function such that

$G_{\overline{1}}^{\mu, \bar{\mu}}(\mu)= \begin{cases}0 & \text { if } \mu<\underline{\mu} \\ 1-\frac{\underline{\mu}-c}{\underline{\mu}} \frac{\mu}{\mu-c} & \text { if } \mu \in[\underline{\mu}, \bar{\mu}) . \\ 1 & \text { if } \mu \geq \bar{\mu}\end{cases}$

We let $G^{\mu, \bar{\mu}}$ denote the corresponding unconditional distribution.

A special property of $G_{1}^{\mu, \bar{\mu}}$ is that it induces unit-elastic demand for the seller. To see this formally, let $\underline{p}(\mu)$ be the inverse function of $\underline{\mu}(p)$, that is, $\mu(1-\underline{p}(\mu))=c$ for all $\mu \in[0,1]$. Then, the probability that the consumer eventually purchases is given by

$\mu_{0}\left(1-G_{\overline{1}}^{\mu, \bar{\mu}}(\underline{\mu}(p))\right)=$

$\begin{cases}\mu_{0} & \text { if } p<\underline{p}(\underline{\mu}) \\ \mu_{0} \frac{\underline{\mu}-c}{\underline{\mu}} \frac{\underline{\mu}(p)}{\underline{\mu}(p)-c}=\mu_{0} \frac{\underline{p}(\underline{\mu})}{p} & \text { if } p \in[\underline{p}(\underline{\mu}), \underline{p}(\bar{\mu})] . \\ 0 & \text { if } p>\underline{p}(\bar{\mu})\end{cases}$

It is then clear that $P S\left(G^{\mu}, \bar{\mu}, p\right)=\mu_{0} \underline{p}(\underline{\mu})$ for all $p \in[\underline{p}(\underline{\mu}), \underline{p}(\bar{\mu})]$ (and $P S\left(G^{\underline{\mu}, \bar{\mu}}, \bar{p}\right)$ $<\mu_{0} \underline{p}(\underline{\mu})$ whenever $\left.p \notin[\underline{p}(\underline{\mu}), \underline{p}(\bar{\mu})]\right)$.

The conditional distribution $G_{1}^{\mu, \bar{\mu}}$ is not necessarily feasible, however. The next lemma shows that $G_{\overline{1}}^{\mu, \bar{\mu}}$ is feasible if and only if $\mu$ is above a certain threshold $\mu^{*}$ and $\bar{\mu}$ is sufficiently close to 1 .

${ }^{6}$ We take the same approach as Roesler and Szentes (2017), who also define and utilize a similar class of distributions. The main difference is that they work with unconditional distributions $G$, not with conditional distributions $G_{1}$. This difference is precisely due to the fundamental distinction between experience and search goods. 
LEMMA 2: There exists $\mu^{*} \in\left(c, \mu_{0}\right)$ and $a$ strictly decreasing function $\phi:\left[\mu^{*}, 1\right) \rightarrow[0,1]$ such that $G_{1}^{\mu, \bar{\mu}}$ is feasible if and only if $\underline{\mu} \geq \mu^{*}$ and $\bar{\mu} \in[\phi(\underline{\mu}), 1]$.

\section{PROOF:}

It can be directly shown that $G_{1}^{\mu, \bar{\mu}}$ increases in the sense of first-order stochastic dominance as either $\mu$ or $\bar{\mu}$ increases. The monotonicity result (that $G_{1}^{\mu}, \bar{\mu}$ is feasible if and only if both $\underline{\mu}$ and $\bar{\mu}$ are above certain thresholds) then follows from Lemma 1. See the online Appendix for a proof that $\mu^{*} \in\left(c, \mu_{0}\right)$ and $\phi$ is strictly decreasing in $\underline{\mu}$.

An important implication of this result is that, since $p(\mu)$ is increasing in $\mu, p\left(\mu^{*}\right)$ is the lowest price that can be implemented by a conditional unit-elastic distribution. In fact, as shown shortly, $p\left(\mu^{*}\right)$ is the lowest implementable price by any feasible distribution. In other words, it is impossible to induce the seller to offer a lower price than $\underline{p}\left(\mu^{*}\right)$.

\section{Seller-Worst Signal}

As explained above, given $G_{1}^{\mu, \bar{\mu}}$, the seller's (maximized) profit is equal to $P S\left(G^{\underline{\mu}, \bar{\mu}}, \underline{p}(\underline{\mu})\right)$ $=\mu_{0} \underline{p}(\underline{\mu})$. Therefore, among the class of unit-elastic conditional distributions, the signal that minimizes $\underline{p}(\underline{\mu})$ also minimizes the seller's profit. Since $\bar{p}(\bar{\mu})=(\mu-c) / \mu$ is increasing in $\mu$, the seller's profit is minimized when $\mu=\mu^{*}$. The following result shows that $G^{\mu^{*}, 1}$

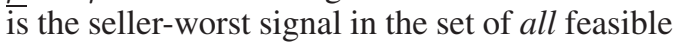
signals.

THEOREM 1: The seller's expected profit is always minimized by $G^{\mu^{*}, 1}$.

\section{PROOF:}

It suffices to show that for any feasible $G_{1}$, there exists a conditional unit-elastic distribution that produces the same profit. Fix any feasible $G$, and denote by $p$ the seller's optimal price under $G$. Let $p^{\prime} \equiv P S(G, p) / \mu_{0}$, and consider $G_{1}^{\mu}\left(p^{\prime}\right), 1$. Clearly, $p^{\prime}$ is optimal to the seller under $G_{1}^{\mu}\left(p^{\prime}\right), 1$, and $P S\left(G_{1}^{\mu}\left(p^{\prime}\right), 1, p^{\prime}\right)=\mu_{0} p^{\prime}=P S(G, p)$. Therefore, it suffices to show that $G_{1}^{\mu}\left(p^{\prime}\right), 1$ is feasible. Since $p$ is the seller's optimal price under $G$, it must be that

$$
\begin{aligned}
P S\left(G, p^{\prime}\right) & =\mu_{0}\left(1-G_{1}\left(\underline{\mu}\left(p^{\prime}\right)\right)\right) p^{\prime} \\
& \leq P S(G, p) \text { for any } p^{\prime} .
\end{aligned}
$$

In addition, by the structure of conditional unit-elastic distributions, we have

$$
\begin{aligned}
P S\left(G^{\underline{\mu}\left(p^{\prime}\right), 1}, p^{\prime}\right) & =\mu_{0}\left(1-G_{1}^{\underline{\mu}(p), 1}\left(\underline{\mu}\left(p^{\prime}\right)\right)\right) p^{\prime} \\
& =\mu_{0} p^{\prime}=\operatorname{PS}\left(G^{\underline{\mu}\left(p^{\prime}\right), 1}, p\right),
\end{aligned}
$$

whenever $p^{\prime} \in[\underline{p}(\underline{\mu}), \underline{p}(1)]$.

These conditions imply that $G_{1}(\mu) \geq G_{1}^{\mu}(p), 1(\mu)$ for all $\mu \geq \underline{\mu}(p)$, that is, $G_{1}^{\underline{\mu}(p), 1}(\mu)$ firstorder-stochastically dominates $G_{1}$. The result then follows from Lemma 1.

For the intuition behind this result, consider the intermediate problem of finding the seller-worst signal among those that induce the same price. Given $p$, the seller's profit is minimized when her demand is minimized. In our search-good problem, this is equivalent to maximizing $G_{1}(\underline{\mu}(p))$, the probability that the consumer does not visit when her true value is 1 . With no other constraint, this is accomplished by inducing only two beliefs, $\mu(p)$ and 1 , in the same way as in the optimal Bayesian persuasion problem (Kamenica and Gentzkow 2011). Under such a signal, however, $p$ is no longer the seller's optimal price, as a slightly lower price would discretely increase demand. In other words, the size of $G_{1}(\underline{\mu}(p))$ is constrained by the fact that $p$ must remain optimal for the seller. A conditional unit-elastic distribution $G_{1}^{\mu, \bar{\mu}}$ is the solution to this constrained optimization problem: if $G_{1}(\underline{\mu}(p))>G_{1}^{\underline{\mu}(p), \bar{\mu}}(\underline{\mu}(p))$, then there necessarily exists $p^{\prime} \quad(<p)$ that gives a higher profit to the seller. This is the fundamental reason why the seller-worst signal is unit-elastic. 


\section{Consumer-Optimal Signal}

Now we characterize the signal that maximizes consumer surplus. We first show that, as for the seller-worst signal, we can restrict attention to conditional unit-elastic distributions.

PROPOSITION 1: If G is feasible and induces the seller to charge $p \in(0,1-c)$, then $G^{\underline{\mu}}(p), 1$ is also feasible and produces larger consumer surplus than $G$, namely $C S\left(G^{\mu}(p), 1, p\right) \geq C S(G, p)$.

\section{PROOF:}

We first show that without loss of generality we can assume that $G$ has no probability mass in $(0, \underline{\mu}(p))$ (i.e., $G(0)=G(\underline{\mu}(p)-))$. Suppose that $G(0)<G(\underline{\mu}(p)-)$. Then, there exists an alternative feasible distribution $G^{\prime}$ that yields the same consumer surplus but has no atom in $(0, \underline{\mu}(p))$. Specifically, define $G^{\prime}$ so that if $\mu \geq \underline{\mu}(p)$, then $G^{\prime}(\mu)=G(\mu)$, while if $\mu<\bar{\mu}(p)$, then

$$
G^{\prime}(\mu)=G(\underline{\mu}(p)-)-\int_{0+}^{\underline{\mu}(p)-} x d G(x) / \underline{\mu}(p) .
$$

In words, $G^{\prime}$ spreads the mass in $(0, \underline{\mu}(p))$ to 0 and $\underline{\mu}(p)$. By construction, $G^{\prime}$ is feasible $\left(\mu_{0}=\bar{\int} \mu d G^{\prime}(\mu)\right)$ and strengthens the seller's incentive to post $p$. Moreover, $C S\left(G^{\prime}, p\right)$ $=C S(G, p)$.

Now suppose that $G$ has no probability mass in $(0, \mu(p))$. Then, by the same argument as in the proof of Theorem 1, we can show that $G_{1}(\mu) \geq G_{1}^{\underline{\mu}}(p), 1(\mu)$ for all $\mu \geq \underline{\mu}(p)$ and, therefore, $G_{1}^{\mu}(p), 1$ first-order-stochastically dominates $G_{1}$. Lemma 1 then implies that if $G$ is feasible, so is $G^{\underline{\mu}}(p), 1$.

In order to show $C S\left(G^{\underline{\mu}}(p), 1, p\right) \geq C S(G, p)$, notice that if $G$ has no probability mass in $(0, \underline{\mu}(p))$ and the seller charges $p$, then consumer surplus can be rewritten as

$$
\begin{aligned}
C S(G, p) & =\mu_{0}(1-p)-(1-G(0)) c \\
= & \mu_{0}(1-p)-\left(1-\left(1-\mu_{0}\right) G_{0}(0)\right) c,
\end{aligned}
$$

as all consumers with value 1 eventually purchase, all consumers with posteriors strictly above 0 search, and $G(0)=\left(1-\mu_{0}\right) G_{0}(0)$ by Bayes' rule. Then, by the above result and Lemma 1,

$$
\begin{gathered}
G_{0}(0)=\Phi\left(G_{1}\right) \leq \Phi\left(G_{\overline{1}}^{\mu, 1}\right)=G_{\overline{1}}^{\mu, 1}(0) \\
\Rightarrow C S(G, p) \leq C S\left(G^{\underline{\mu}(p), 1}, p\right)
\end{gathered}
$$

Clearly, given $G_{1}^{\mu, \bar{\mu}}$, it is optimal for the consumer that the seller charges the lowest optimal price $\underline{p}(\underline{\mu})$. Then, as in the proof of Proposition 1,

$$
\begin{aligned}
C S & \left(G^{\underline{\mu}, \bar{\mu}}, \underline{p}(\underline{\mu})\right) \\
& =\mu_{0}(1-\underline{p}(\underline{\mu}))-\left(1-\left(1-\mu_{0}\right) G_{0}^{\mu, \bar{\mu}}(0)\right) c \\
& =\mu_{0} \frac{\underline{\mu}-c}{\underline{\mu}} \ln \left(\frac{\underline{\mu}}{\underline{\mu}-c} \frac{\bar{\mu}-c}{\bar{\mu}}\right) .
\end{aligned}
$$

The second equality is based on the explicit solution for $G_{0}^{\mu}, \bar{\mu}(0)$, which is obtainable from (2) and the specific structure of $G_{\overline{1}}^{\mu, \bar{\mu}}$. Clearly, $C S\left(G^{\underline{\mu}}, \bar{\mu}, \underline{p}(\underline{\mu})\right)$ is always increasing in $\bar{\mu}$. Since increasing $\bar{\mu}$ also slackens the feasibility constraint (Lemma 2), we can restrict attention to the case where $\bar{\mu}=1$. This implies that the problem reduces to identifying the value of $\underline{\mu}$ that maximizes

$$
\begin{aligned}
& C S\left(G^{\underline{\mu}, 1}, \underline{p}(\underline{\mu})\right) \\
& \quad=\mu_{0} \frac{\underline{\mu}-c}{\underline{\mu}} \ln \left(\frac{\underline{\mu}}{\underline{\mu}-c}(1-c)\right) .
\end{aligned}
$$

Based on this observation, the following result fully characterizes the consumer-optimal distribution.'

THEOREM 2: Let $c^{*} \equiv \mu_{0}(e-2) /\left(e-2 \mu_{0}\right)$ and $\mu^{* *} \equiv e c /(e-1+c)$. If $c \leq c^{*}$, then $G^{\mu^{*}, 1}$ maximizes consumer surplus, while if $c \in\left(c^{*}, \mu_{0}\right)$, then $G^{\mu^{* *}, 1}$ maximizes consumer surplus.

\footnotetext{
${ }^{7}$ We make an implicit assumption that under the consumer-optimal signal, the seller offers the lowest price among all optimal prices. Note that this assumption is not necessary for the seller-worst signal, and if this assumption fails, then the consumer-optimal signal does not exist.
} 
PROOF:

$C S\left(G^{\underline{\mu}, 1}, \underline{p}(\underline{\mu})\right)$ in (4) is single-peaked in $\underline{\mu}$ and maximized when $\underline{\mu}=\mu^{* *}$. This means that if $\mu^{*} \geq \mu^{* *}$, then $\partial C S\left(G^{\underline{\mu}, 1}, \underline{p}(\underline{\mu})\right) / \partial \underline{\mu} \leq 0$ for all $\underline{\mu} \geq \mu^{*}$ and, therefore, $C S\left(G^{\underline{\mu}, 1}, \underline{p}(\underline{\mu})\right)$ is maximized when $\underline{\mu}=\mu^{*}$. Otherwise, $\operatorname{CS}\left(G^{\underline{\mu}, 1}, \underline{p}(\underline{\mu})\right)$ is maximized when $\underline{\mu}=\mu^{* *}$.

It remains to show that $\mu^{*} \geq \mu^{* *}$ if and only if $c \leq c^{*}$. Recall that $\mu^{*}$ satisfies

$$
\begin{aligned}
\Phi\left(G_{1}^{\mu^{*}, 1}\right) & =-\frac{\mu_{0}-\mu^{*}}{\mu^{*}\left(1-\mu_{0}\right)} \\
+ & \frac{\mu_{0}}{1-\mu_{0}} \frac{\mu^{*}-c}{\mu^{*} c} \ln \left(\frac{\mu^{*}(1-c)}{\mu^{*}-c}\right)=0 .
\end{aligned}
$$

The desired result can be obtained from this implicit function by recognizing that $c^{*}$ is the unique cost in $\left(0, \mu_{0}\right)$ at which $\mu^{*}=\mu^{* *}$ and showing that when $c=c^{*}$,

$$
\begin{aligned}
\frac{d \mu^{* *}}{d c} & =\frac{(e-1)\left(e-2 \mu_{0}\right)^{2}}{e\left(e-\mu_{0}-1\right)^{2}} \\
& >\frac{\left(e-2 \mu_{0}\right)^{2}}{e\left(e-\mu_{0}-1\right)^{2}}=\frac{d \mu^{*}}{d c} .
\end{aligned}
$$

The consumer-optimal signal has two notable features. First, it induces excessive search: the consumer pays to visit even if her true value is 0 and, therefore, she will not purchase for sure. This is obviously detrimental to consumer surplus ex post but necessary to improve consumer surplus ex ante: if the consumer visits only when her true value is 1 (i.e., $\mu=1$ ), then the seller charges $1-c$ and, therefore, leaves no consumer surplus. In other words, ex post regret is unavoidable to reduce the seller's price. Note that this is similar to the result in Roesler and Szentes (2017) that the consumer-optimal signal induces the consumer to purchase even if her true value falls short of the price.

Second, the signal that minimizes the seller's price, $G^{\mu^{*}, 1}$, is not necessarily the consumeroptimal signal. This is because the consumeroptimal signal must balance inducing a lower price and reducing search expenditures. If $c$ is rather small, then minimizing the seller's price is more beneficial to the consumer than avoiding excessive search and, therefore, $\underline{\mu}=\mu^{*}$ is optimal. If $c$ is sufficiently large, however, the marginal cost of excessive search outweighs the corresponding marginal benefit (of further lowering the price). In this case, it is beneficial to discourage the consumer from visiting by giving some mass of $v=0$ a posterior of 0 , which comes at the cost of increasing $\underline{\mu}$ and therefore $p$ as well. This makes the consumer-optimal signal differ from the signal that minimizes the seller's price. Clearly, this divergence between the seller-worst signal and the consumer-optimal signal stems from the unique nature of search goods: in Roesler and Szentes (2017) (and also in other related studies), they always coincide.

\section{Conclusion}

In this paper, we characterize the seller-worst signal and the consumer-optimal signal for the monopoly pricing problem with search goods. Our results complement Anderson and Renault (2006), who characterize the seller-optimal signal for the same environment, and Roesler and Szentes (2017), who study the experience-good counterpart to our problem. Whereas we think that this specific contribution is not marginal, our main aim is to bring attention to search goods, which have received disproportionately less attention than experience goods from the recent literature on information design. Our analysis takes the natural first step toward incorporating search goods into information design. We think that there are many interesting information-design problems for search goods, as there have been for experience goods.

\section{REFERENCES}

-Anderson, Simon P., and Régis Renault. 2006. "Advertising Content." American Economic Review 96 (1): 93-113.

Au, Pak Hung. 2018. "Competition in Designing Pandora's Boxes." https://papers.ssrn. com/sol3/papers.cfm?abstract_id=3141387 (accessed February 20, 2019).

- Bergemann, Dirk, Benjamin Brooks, and Stephen Morris. 2015. "The Limits of Price Discrimination." American Economic Review 105 (3): 921-57. 
Bergemann, Dirk, Benjamin Brooks, and Stephen Morris. 2017. "First-Price Auctions with General Information Structures: Implications for Bidding and Revenue." Econometrica 85 (1): 107-43.

Bergemann, Dirk, and Martin Pesendorfer. 2007. "Information Structures in Optimal Auctions." Journal of Economic Theory 137 (1): 580-609.

Hinnosaar, Toomas, and Keiichi Kawai. 2018. "Robust Pricing with Refunds." https:// papers.ssrn.com/sol3/papers.cfm?abstract id=3165146 (accessed February 20, 2019).

Hu, Ju, and Mustafa Dogan. 2018. "Consumer Search and Optimal Information." https:// papers.ssrn.com/sol3/papers.cfm?abstract_ id=3308157 (accessed February 20, 2019).

Hulko, Artem, and Mark Whitmeyer. 2018. "Information Provision in a Sequential Search Setting." https://arxiv.org/pdf/1802.09396.pdf (accessed February 20, 2019).

-Kamenica, Emir, and Matthew Gentzkow. 2011. "Bayesian Persuasion." American Economic Review 101 (6): 2590-2615.

Nelson, Phillip. 1970. "Information and Consumer Behavior." Journal of Political Economy 78 (2): 311-29.

- Roesler, Anne-Katrin, and Balázs Szentes. 2017. "Buyer-Optimal Learning and Monopoly Pricing." American Economic Review 107 (7): 2072-80. 